\title{
Correcting Wavefront Distortion of Dual-Wavelength Beams Due to Atmospheric Turbulence with a Correction Coefficient
}

\author{
Xizheng Keㄹ, Xiaozhan Chen ${ }^{2 *}$ \\ ${ }^{1}$ Faculty of Automation and Information Engineering, Xi' an University of Technology, Xi'an, China \\ ${ }^{2}$ Shaanxi Civil-Military Integration Key Laboratory of Intelligence Collaborative Networks, Xi'an, China \\ Email: *xiaozhan.chen@foxmail.com
}

How to cite this paper: Ke, X.Z. and Chen, X.Z. (2020) Correcting Wavefront Distortion of Dual-Wavelength Beams Due to Atmospheric Turbulence with a Correction Coefficient. Optics and Photonics Journal, 10, 64-77.

https://doi.org/10.4236/opj.2020.104006

Received: February 26, 2020

Accepted: April 20, 2020

Published: April 23, 2020

Copyright $\odot 2020$ by author(s) and Scientific Research Publishing Inc. This work is licensed under the Creative Commons Attribution International License (CC BY 4.0).

http://creativecommons.org/licenses/by/4.0/

(c) (i) Open Access

\begin{abstract}
In order to improve the correction effect of the adaptive optical system in coherent optical communication, we investigate the relative distortion between the wavefronts of different wavelengths of the beams transmitted on the near-ground horizontal atmospheric turbulent links emitted by coherent optical communication system. And the situation is analyzed when the wavelength corresponding to the wavefront detected by the wavefront detector and the wavelength corrected by the deformed mirror are different, the influence of the wavelength factor on the adaptive optical system correction. We use a series of trigonometric functions and the Hankel transformation to derive the corrected residual variance and the Strehl ratio between the wavefront distortions of the wavelengths of the dual-wavelength combined beam in atmospheric turbulence. In relation to the parameters of the turbulent environment, the ensemble average of the wavefront difference corresponding to different wavelengths the derived is proposed as the coefficient to correct the dual-wavelength adaptive optical system. The results show that the statistic of the turbulence internal scale has a major influence on the difference between the wavefronts. By adding the correction coefficient, the signal light's wavefront of the coherent optical communication system can be corrected more effectively by the dual-wavelength adaptive optical closure.
\end{abstract}

\section{Keywords}

Coherent Optical Communication, Dual-Wavelength Wavefront, Atmospheric Turbulence, Adaptive Optics System, Wavefront Residual Variance 


\section{Introduction}

The coherent optical communication system uses a laser beam as information carrier to communicate between channels in space (including the near-Earth atmosphere, low orbit, medium orbit, synchronous orbit, interstellar space), it based on coherent detection has the advantages of high receiving sensitivity, strong anti background noise and so on. The most fundamental effect of atmospheric turbulence for the channel on the laser transmission is the degradation of the coherence of the transmitted beam. The random change of atmospheric pressure and density caused by the random change of atmospheric temperature leads to the random change of atmospheric refractive index. When the beam is transmitted in an area with the random change of the refractive index, an optical path difference is generated, which leads to the wavefront distortion. The wavefront distortion makes the beam energy of the beam irradiating the receiving end sensor be unevenly distributed, which seriously affects the received power of the signal light and reduces the communication quality of the system [1]. In order to suppress the influence of atmospheric turbulence on the laser beam, adaptive optics technology can effectively overcome the wavefront distortion caused by atmospheric turbulence. Adaptive optics ( $\mathrm{AO})$ is a technology that uses deformable mirrors to correct the distortion of optical wavefront caused by atmospheric jitter, so as to improve the performance of the optical system. Adaptive optics first detects the distortion of a wavefront through the wavefront sensor, and then corrects the wavefront in real-time through a small deformable mirror, several actuators are installed after the deformable mirror to support the movement of the deformable mirror. As shown in Figure 1, the coherent optical communication system uses a laser beam as the beacon light, which is coaxial with a wavelength different from that of the signal light, and the adaptive optics system on the coherent optical communication receiving end uses the beacon's optical wavefront slope for wavefront reconstruction-the reconstruction information is applied to the deformable mirror to correct the wavefront of the signal light. Correcting the wavefront of the communication beam in this technique increases the received light power and improves the quality of the communication [2] [3] [4]. Different wavelengths of light have different fluctuations of the refractive index while in the same transmission path, and different wavelengths are diffracted at different propagation rates, when the lights through the same transmission path, the detected and corrected wavefronts have spatial differences [5]

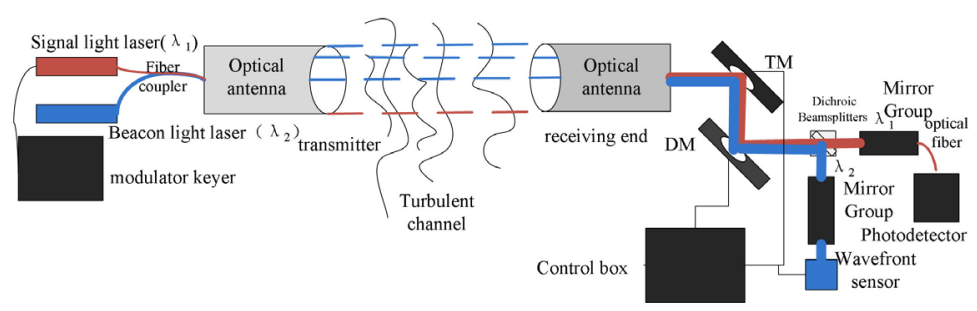

Figure 1. Coherent optical communication system and adaptive optics. 
[6] [7], the study of this difference is of great significance to the optimization of system performance.

Winocur [8] used the time power spectral density of Zernike polynomial coefficients to study the wavefront difference and residual error of the adaptive optics of dual-wavelength correction. Hogge [9] used the perturbation method to derive the wavefront difference between the two-wavelength plane wave and the spherical wave. Lukin [10] also used the same method to derive the function of the wavefront structure function and variance of the dual-wavelength Gaussian beam, and proposed their application range. In the literature [11] two ways of correcting dual-wavelength adaptive optics were studied (1) using a reference source correction and (2) using a reflection source correction. Ishimaru [12] used the correlation function of the log amplitude of the dual-wavelength beam and the time power spectral density of the phase to analyze the temporal correlation of the wavefront of the dual-wavelength beam. Gorelaya et al. [13] conducted a wavefront measurement experiment in an indoor turbulence pool with dual-wavelength beam transmission on the same transmission path. The measurement results show that the wavefront distortion of the short-wavelength beam is greater than that of the long-wavelength beam under the same turbulent environment. Lee et al. [14] constructed a turbulent phase screen to simulate the wavefront transmission of a dual-wavelength beam and analyzed the wavefront fluctuation of the dual-wavelength beam by statistical methods. Feng et al. [15] derived the correlation function of the Zernike polynomial coefficients of the wavefront of the two-wavelength beam, analyzed the correlation of the wavefront of the two-wavelength beam at different Zernike orders, and proposed a method for reducing the residual error of the corrected wavefront.

This paper studies the relative variation between the wavefront distortions of the two-wavelength combined beam in horizontal transmission and the influence of wavelength factor on the adaptive optical system. In the first part, we derive the residual variance and Strehl ratio of the wavefront correction of the dual-wavelength beam with a circular aperture in the same transmission path. In the second part, we study the effects of the scale of the turbulence, and transmission distance on the residual wavefront variance that correction of the adaptive optical system of tow wavelengths. We also study the ratio of the two wavefronts fluctuation variances of the dual-wavelength beam in the third part, the role of this ratio is important for improving the quality the correction to the dual-wavelength adaptive optics closed-loop and the improving the signal light's power received by the system in multi-wavelength laser communication systems.

\section{Theoretical Analysis}

\subsection{Residual Error of the Combined Wavefront Correction}

The transmitting end of the coherent optical communication system emits two beams of different wavelengths. One is the beacon beam and the other the signal beam, two beams of light are emitted in the optical antenna and become com- 
bined beams. The combined beam passes through the same horizontal atmospheric turbulence path and arrives at the adaptive optics module in the receiving end of the system. The wavefront of the signal wavelength $\lambda_{1}$ is compensated by the wavefront compensation of the beacon wavelength $\lambda_{2}$ in the module. When the beam travels along the $\mathrm{z}$ axis and enters the aperture of radius $\mathrm{R}$, Wavefront of $\lambda_{1}$ and $\lambda_{2}$ in the aperture showed in Figure 2. $\rho_{1}$ is the point of wavefront with $\lambda_{1}, \rho_{2}$ is the point of wavefront with $\lambda_{2}, \rho$ is the distance between $\rho_{1}$ and $\rho_{2}$. The compensated residual wavefront variance can be expressed by Equations (1), (2), (3), and (4), which also represent the difference between the wavefront aberrations corresponding to the two wavelengths of horizontal transmission.

$$
\begin{gathered}
\Delta=\frac{1}{\pi R^{2}} \iint w(\rho)\left(\varphi_{\lambda_{1}}(\rho)-\varphi_{\lambda_{2}}(\rho)\right)^{2} \mathrm{~d}^{2} \rho \\
w(\rho)=\left\{\begin{array}{l}
1, \rho<R \\
0, \rho>R
\end{array}\right. \\
\left\langle\varphi_{\lambda i}\left(k_{i}, \rho\right)\right\rangle^{2}=4 \pi^{2} \int_{0}^{L} \mathrm{~d} z \int_{0}^{\infty} \kappa \mathrm{d} \kappa \Phi_{n}(\kappa) J_{0}(\rho \kappa) \cos ^{2}\left(\frac{(L-Z) \gamma_{i} \kappa^{2}}{2 k_{i}}\right), i=1,2 \\
\left\langle\varphi_{\lambda_{1}}\left(k_{1}, \rho\right) \varphi_{\lambda_{2}}\left(k_{2}, \rho\right)\right\rangle \\
=4 \pi^{2} \int_{0}^{L} \mathrm{~d} z \int_{0}^{\infty} \kappa \mathrm{d} \kappa \Phi_{n}(\kappa) J_{0}(\rho \kappa) \cos \left(\frac{(L-Z) \gamma_{1} \kappa^{2}}{2 k_{1}}\right) \cos \left(\frac{(L-Z) \gamma_{2} \kappa^{2}}{2 k_{2}}\right)
\end{gathered}
$$

where $\varphi_{\lambda_{1}}$ represents the signal wavefront on the receiving aperture, $\varphi_{\lambda_{2}}$ represents the beacon wavefront, $w(\rho)$ is the aperture function, $R$ is the aperture radius, and $\rho$ is the distance between the two points on the beam section, it is also the radius of aperture the beam enters. And where $k_{1}$ is the wave number of signal waves, $k_{2}$ is the wave number of beacons, $J_{0}$ is the zero-order Bessel function, and $L$ is the propagation distance. The Gaussian beam can be approximated as a plane wave after long-distance transmission, and $\gamma_{i}$ is one [16]. Equation (1) is expanded, such that the ensemble average of the wavefront variance corresponding to a single wavelength can be written as Equation (3), and the wavefront correlation function corresponding to $\lambda_{1}$ and $\lambda_{2}$ can be written as Equation (4) [16]. Equations (3) and (4) are derived by series approximation and Hankel transform [17], and Equation (5) gives the power spectrum in the inertial sub region described by the Kolmogorov turbulence theory model [18]:

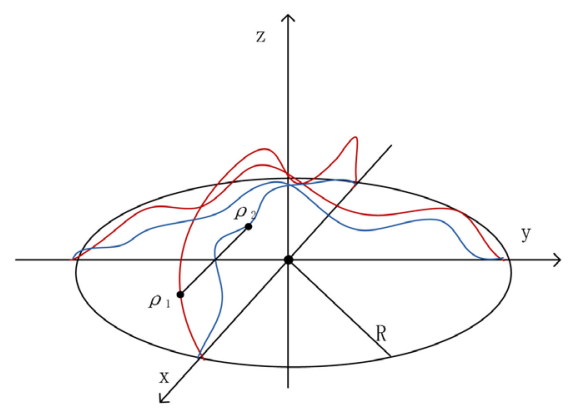

Figure 2. Wavefront of $\lambda_{1}$ and $\lambda_{2}$ in aperture. 


$$
\Phi_{n}(k)=0.033 C_{n}^{2} \kappa^{-11 / 3}
$$

Equation (6) gives the ensemble average of wavefront variance and Equation (7) gives the wavefront correlation function corresponding to the derived wavelengths. $L_{0}$ is the outer scale of turbulence and $l_{0}$ is the inner scale of turbulence, where $\mathrm{Q}$ is $0.132 \pi^{2} C_{n}^{2}$.

$$
\begin{aligned}
& \left\langle\varphi_{\lambda_{i}}\left(k_{i}, \rho\right)\right\rangle^{2} \\
= & Q\left\{\begin{array}{l}
\frac{2^{-\frac{2}{6}} L}{\rho^{-\frac{8}{3}}} \frac{\Gamma\left(-\frac{5}{6}\right)}{\Gamma\left(\frac{17}{6}\right)}-\frac{L^{3}}{12 k_{i}^{2}} \frac{2^{\frac{4}{3}}}{\rho^{\frac{4}{3}}} \frac{\Gamma\left(\frac{7}{6}\right)}{\Gamma\left(\frac{5}{6}\right)}, \rho \neq 0 \\
-\frac{3 L}{5}\left[\left(\frac{2 \pi}{L_{0}}\right)^{-5 / 3}-\left(\frac{2 \pi}{l_{0}}\right)^{-5 / 3}\right]-\frac{2 L^{3}}{56 k_{i}^{2}}\left[\left(\frac{2 \pi}{L_{0}}\right)^{7 / 3}-\left(\frac{2 \pi}{l_{0}}\right)^{7 / 3}\right], \rho=0 \\
\left\langle\varphi_{\lambda_{1}}\left(k_{1}, \rho\right) \varphi_{\lambda_{2}}\left(k_{2}, \rho\right)\right\rangle \\
L \frac{2^{-8 / 3} \frac{\Gamma(-5 / 6)}{\rho^{-8 / 3}}-\frac{7 L^{3}}{3} \frac{k_{1}^{2}+k_{2}^{2}}{8 k_{1}^{2} k_{2}^{2}} \frac{2^{4 / 3}}{\rho^{4 / 3}} \frac{\Gamma(7 / 6)}{\Gamma(-5 / 6)}, \rho \neq 0}{3 L}\left[\left(\frac{2 \pi}{L_{0}}\right)^{-5 / 3}-\left(\frac{2 \pi}{l_{0}}\right)^{-5 / 3}\right]-\frac{L^{3}\left(k_{1}^{2}+k_{2}^{2}\right)}{28 k_{1}^{2} k_{2}^{2}}\left[\left(\frac{2 \pi}{L_{0}}\right)^{7 / 3}-\left(\frac{2 \pi}{l_{0}}\right)^{7 / 3}\right], \rho=0
\end{array}\right.
\end{aligned}
$$

When the wavefront of the beam with the wavelength $\lambda_{2}$ is used to compensate for the wavefront of the beam with the wavelength $\lambda_{1}$, the residual variance of the two spatial points on the aperture of a circle with radius $R$ at a distance of $\rho$ can be written as Equation (8):

$$
\begin{aligned}
\Delta= & \frac{4 \pi}{R^{2}} \iint w(\rho)\left\{\int _ { 0 } ^ { L } z \mathrm { d } z \int _ { 0 } ^ { \infty } \kappa \mathrm { d } \kappa \Phi _ { n } ( \kappa ) J _ { 0 } ( \rho \kappa ) \left[\cos \left(\frac{(L-Z) \kappa^{2}}{2 k_{1}}\right)\right.\right. \\
& \left.\left.-\cos \left(\frac{(L-Z) \kappa^{2}}{2 k_{2}}\right)\right]^{2}\right\} \mathrm{d}^{2} \rho
\end{aligned}
$$

By the same method, the residual variance of the two-wavelength wavefront correction of the horizontally transmitted beam is derived as Equation (9), (10), with $\mathrm{c}=7.1962 \mathrm{e}-05$.

$$
\begin{aligned}
& \Delta=\frac{4 \pi}{R^{2}} \iint w(\rho)\left\{\frac{\left.2^{16 / 3} \Gamma\left(\frac{19}{6}\right) C_{n}^{2} \int^{L}(L-Z)^{4} \mathrm{~d} z\right\} \mathrm{d}^{2} \rho}{\rho^{16 / 3} \Gamma\left(-\frac{7}{6}\right)} \int_{0}^{2}\right. \\
& \Delta=\left\{\begin{array}{l}
\frac{39.4 \pi}{R^{2}} L^{5} C_{n}^{2} \frac{\left(k_{1}^{2}-k_{2}^{2}\right)^{2}}{k_{1}^{4} k_{2}^{4}} \frac{L_{0}^{\frac{19}{3}}-l_{0}^{\frac{19}{3}}}{\left(L_{0} l_{0}\right)^{\frac{19}{3}}}, \rho=0 \\
\mathrm{c} \frac{\pi}{R^{2}} \frac{\Gamma\left(\frac{19}{6}\right) L^{5}}{\rho^{-10 / 3} \Gamma\left(-\frac{7}{6}\right)} C_{n}^{2} \frac{\left(k_{1}^{2}-k_{2}^{2}\right)^{2}}{k_{1}^{4} k_{2}^{4}}, l_{0} \leq \rho \leq L_{0}
\end{array}\right.
\end{aligned}
$$


When the distances $\rho$ between the point $\rho_{1}$ and $\rho_{2}$ on the aperture of a circle with radius $R$ are zero, means on the same point, the value of the Bessel function in the Equation (8) is one, the applicable range of the Kolmogorov turbulence spectrum is in the inertia sub-region, so the integral range of the spatial wavenumber should be changed from $2 \pi / l_{0}$ to $2 \pi / L_{0}$. Re-integrate, and then obtain the two-wavelength wavefront corrected residual variance when $\rho=0$. The Strehl ratio is calculated using the corrected residual variance. The Strehl ratio can then be used to evaluate the wavefront quality after wavefront correction in the dual-wavelength adaptive optics system. The reason for the correction in the residual variance is that each actuator of the deformable mirror moves according to the reconstructed wavefront $\varphi_{\lambda_{2}}$ when the deformable mirror corrects the wavefront $\varphi_{\lambda_{1}}$, as shown in Figure 3(a). The ideal surface shape of the deformable mirror is a conjugate of $\varphi_{\lambda_{2}}$; thus, the theoretical correction to the residual variance is achieved. In practice, the deformable mirror, which is composed of several segmented sub-reflect mirrors supported by moving actuators, like the 4 $\times 4$ deformable mirror in Figure 3(b) and Figure 3(c), cannot correct wavefront by point-to-point in finite correction space. When $\rho$ is smaller than the size of the sub reflector, the local wavefront of two points with wavefront difference will receive the same amount of correction, so that the wavefront difference corresponding to the two different wavelengths is not corrected. When $\lambda_{1}$ is $1.5 \mu \mathrm{m}$ and $\rho$ is zero, the corrected residual variance varies with wavelength $\lambda_{2}$ as shown in Figure 4.

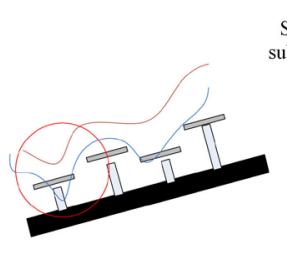

(a)

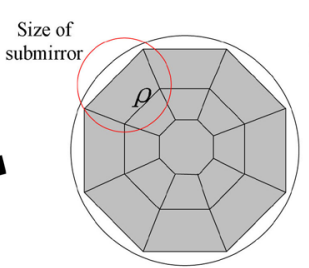

(b)

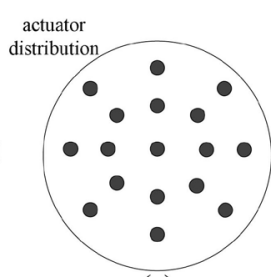

(c)

Figure 3. If it is a sub-mirror distribution of a $4 \times 4$ reflective phase change mirror, the size of the sub-mirror has an important influence on the correction of the wavefront corresponding to the wavelength in the region.

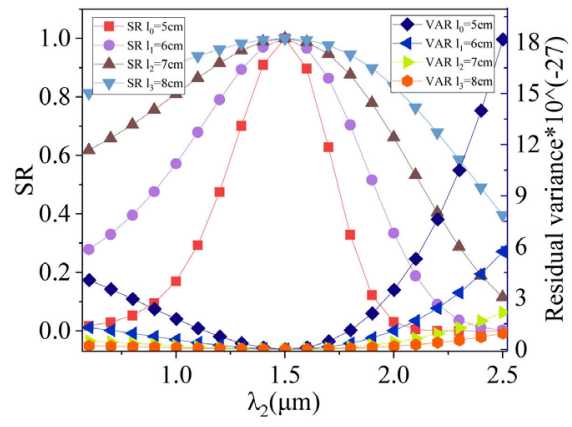

(a)

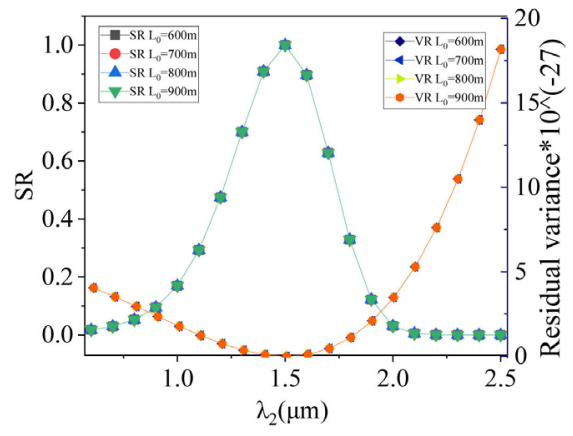

(b)

Figure 4. Variation of corrected residual variance and Strehl ratio when $\lambda_{1}$ is 1.5 microns (a) when the outer scale is $500 \mathrm{~m}$; (b) when the inner scale is $5 \mathrm{~cm}$. 
With the change of $\lambda_{2}$ and the difference between the internal scale and the external scale, the trend of the corrected residual variance and the Strehl ratio at the same point between the two wavefronts after horizontal transmission for 10 $\mathrm{km}$ is shown in Figure 4. To better represent the trend of the change, the variance was scaled up. As the internal scale of atmospheric turbulence increases and $\lambda_{2}$ comes closer to $\lambda_{1}$, the two wavefront differences and the corrected residual variance gradually decrease, and the Strehl ratio gradually increases. In Figure 4(b) image with the same internal scale, the corrected residual variance and the Strehl ratio have the same trend as that shown in Figure 4(a) image when the two wavelengths are closer. With the increase of the external scale, the influences of the corrected residual variance and the Strehl ratio are not obvious, and the curves almost coincide. The analysis shows that the internal scale has a relatively large influence on the difference between the two wavefront fluctuations relative to the external scale.

Figure 5(a) shows the two points $\rho_{1}$ and $\rho_{2}$ in the aperture at a distance $\rho$ of $10 \mathrm{~cm}$ when the two wavelengths are fixed, the corrected residual variance without the correction coefficient and the Strehl ratio changes with the transmission distance under different turbulent intensities. The corrected residual variance continues to increase as the transmission distance increases, the greater the turbulence intensity, the faster the increased speed, and the corrected Strehl ratio gradually decreases with the increase of the transmission distance, and the decrease slows down. Figure 5(b) shows that when the transmission distance and the turbulence intensity are constant, the corrected residual variance without the correction coefficient varies with $\lambda_{2}$ at different distances of two points. As $\lambda_{2}$ approaches $\lambda_{1}$ and the distance between the point $\rho_{1}$ and $\rho_{2}$ gradually decreases, the Strehl ratio gradually increases and then decreases as the difference between the two wavelengths increases. The smaller the distance between the point $\rho_{1}$ and $\rho_{2}$ of the wavefront in the aperture, the faster the Strehl ratio rises.

In the process of horizontal transmission, the internal scale has a greater effect on the two wavefronts with different wavelengths than the size of the turbulent outer scale. When the internal scale increases, the turbulence is generally

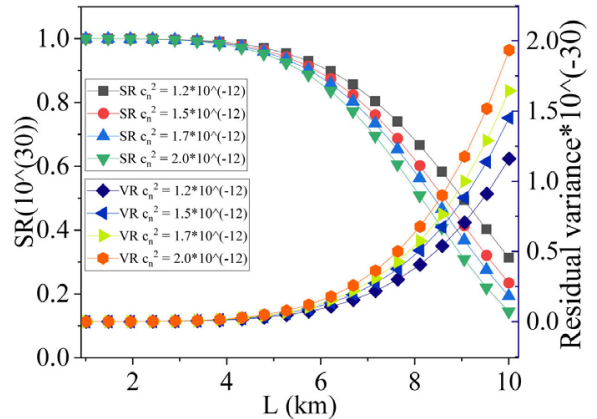

(a)

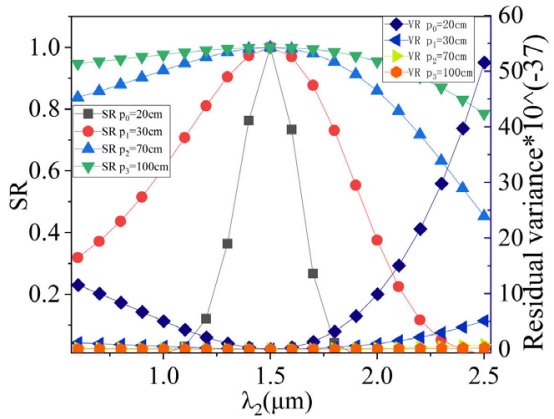

(b)

Figure 5. Variation of the two-wave variance with center $\rho$. (a) Two-wave variance with transmission distance and refractive index structure constant. (b) Two-wave corrected residual variance and Strehl ratio with $\lambda_{2}$ and two-point distance. 
stronger and has a greater impact on the Strehl ratio after dual-wavelength correction. The influence of the changes of external scale on the correction with wavefront of different wavelengths is very small, and the smaller the degree difference between the two wavelengths, the more two wavefronts tend to be the same spatial distribution at the same point in the aperture.

\subsection{Wavefront Residual Error with Correction Coefficient}

To reduce the residual variance of the wavefront $\varphi_{\lambda_{1}}$, when the wavefront $\varphi_{\lambda_{2}}$ of the beacon beam detected in wavefront sensor, the ratio of the ensemble average of wavefront standard deviation of corresponding two wavelengths in Equation (3) is taken as the correction coefficient. That is $\varphi_{\lambda_{1}}-G \varphi_{\lambda_{2}}$, so the corrected residual variance containing the correction coefficient can be written as Equation (11).

$$
\Delta_{2}=\frac{1}{\pi R^{2}} \iint w(\rho)\left(\varphi_{\lambda_{1}}(\rho)-G \varphi_{\lambda_{2}}(\rho)\right)^{2} \mathrm{~d}^{2} \rho
$$

$G$ can be written as Equation (12), $C_{n 2}^{2}$ is the refractive index structure constant corresponding to $\lambda_{1}, C_{n 2}^{2}$ is the refractive index structure constant corresponding to $\lambda_{2}$, and $A, B, C$, and $D$ are environmental factors given by Equations (13), (14), (15), and (16):

$$
\begin{aligned}
& G=\left\langle\frac{\varphi_{\lambda_{1}}\left(k_{1}, \rho\right)}{\varphi_{\lambda_{2}}\left(k_{2}, \rho\right)}\right\rangle=\left\{\begin{array}{l}
\sqrt{\frac{C_{n 1}^{2}\left(1-\frac{B}{A} k_{2}^{2}\right)}{C_{n 2}^{2}\left(1-\frac{B}{A} k_{1}^{2}\right)}}, \rho \neq 0 \\
\sqrt{\frac{C_{n 1}^{2}\left(C-\frac{1}{k_{1}^{2}} D\right)}{C_{n 2}^{2}\left(C-\frac{1}{k_{2}^{2}} D\right)}}, \rho=0
\end{array}\right. \\
& A=3 \times 2^{\frac{5}{3}} L \rho^{\frac{4}{3}} k_{1}^{2} k_{2}^{2} \Gamma\left(-\frac{5}{6}\right) \Gamma\left(\frac{5}{6}\right) \\
& B=2^{\frac{4}{3}} L^{3} \rho^{-\frac{8}{3}} \Gamma\left(\frac{17}{6}\right) \Gamma\left(\frac{7}{6}\right) \\
& C=-\frac{3}{5} L\left[\left(\frac{2 \pi}{L_{0}}\right)^{-5 / 3}-\left(\frac{2 \pi}{l_{0}}\right)^{-5 / 3}\right] \\
& D=\frac{L^{3}}{28}\left[\left(\frac{2 \pi}{L_{0}}\right)^{-7 / 3}-\left(\frac{2 \pi}{l_{0}}\right)^{-7 / 3}\right]
\end{aligned}
$$

On the horizontal link near the ground, the refractive index structure constant is generally considered to be the constant corresponding to the environment. It can be calculated in the range of a given band (17), out scale can be calculated in Equation (18) [19], and the method of literature [20] can be used to determine the internal scale. $G$ is brought into Equation (11) and the same method is used 
to derive Equations (19) and (20).

$$
\begin{gathered}
C_{n}^{2}=\left[\frac{77.6 \mathrm{e}-16}{T^{2}}\left(1+(7.52 \mathrm{e}-3) \lambda^{-2}\right) P\right]^{2} C_{T}^{2} \\
L_{0}=0.5+5 \exp \left[-\left(\frac{z-7500}{2500}\right)^{2}\right] \\
\Delta_{2}=\frac{0.132 \pi C_{n}^{2}}{R^{2}}\left\{-\frac{1}{2} L\left[\left(\frac{2 \pi}{L_{0}}\right)^{-2}-\left(\frac{2 \pi}{l_{0}}\right)^{-2}\right]\right. \\
+\frac{(4.19 \mathrm{e}-3) L^{5}\left(k_{2}^{2}-G k_{1}^{2}\right)^{4}}{k_{1}^{4} k_{2}^{4}} \times\left[\left(\frac{2 \pi}{L_{0}}\right)^{19 / 3}-\left(\frac{2 \pi}{l_{0}}\right)^{19 / 3}\right] \\
\left.-\left(\frac{-L^{3}}{7}\right) \frac{(1-G)\left(k_{2}^{2}-G k_{1}^{2}\right)}{k_{1}^{2} k_{2}^{2}}\left[\left(\frac{2 \pi}{L_{0}}\right)^{7 / 3}-\left(\frac{2 \pi}{l_{0}}\right)^{7 / 3}\right]\right\}, \rho=0 \\
\Delta_{2}=\frac{0.132 \pi^{2} C_{n}^{2}}{R^{2}}\left[\frac{L 2^{-8 / 3}}{\rho^{-8 / 3}} \frac{\Gamma(5 / 6)}{\Gamma(17 / 6)}+\frac{17 L^{5} 2^{16 / 3}}{640 \rho^{16 / 3}} \frac{\Gamma(19 / 6)\left(k_{2}^{2}-G k_{1}^{2}\right)^{4}}{\Gamma(-7 / 6) k_{1}^{4} k_{2}^{4}}\right. \\
\left.-\left(-L^{3}\right) \frac{2^{4 / 3}}{24 \rho^{4 / 3}} \frac{\Gamma(7 / 6)}{\Gamma(5 / 6)} \frac{(1-G)\left(k_{2}^{2}-G k_{1}^{2}\right)}{k_{1}^{2} k_{2}^{2}}\right], l_{0} \leq \rho \leq L_{0}
\end{gathered}
$$

If the transmission distance is $10 \mathrm{~km}$ and $\lambda_{1}$ is $1.5 \mu \mathrm{m}$, then for different $\lambda_{2}$ the corresponding correction coefficients are calculated according to Equations (19) and (20), and the correction residual variance and Strehl ratio containing the correction coefficients are obtained. The change of the correction residual variance and Strehl ratio with $\lambda_{2}$ with the correction coefficient at the same point that $\rho$ equal to zero as in Figure 4 is shown in Figure 6(a). When the external scale is $50 \mathrm{~cm}$, and $\lambda_{2}$ is less than $\lambda_{1}$ for different internal scales, the larger the internal scale is, the faster the correction residual variance will decrease. At the same time the faster the corrected Strehl ratio will rise, and the correction effect is significantly better than that shown in Figure 3 . The corrected residual variance with the correction coefficient at the distance $\rho$ and the change of the Strehl

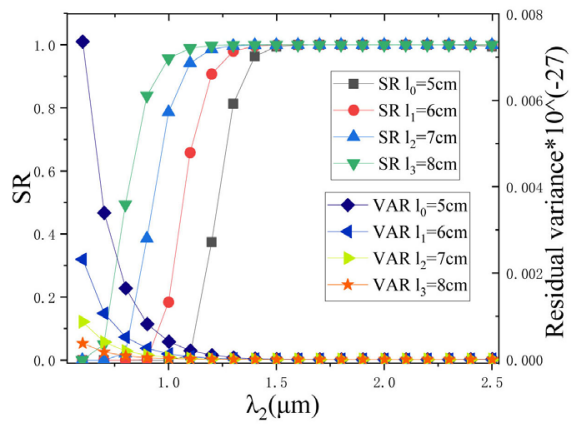

(a)

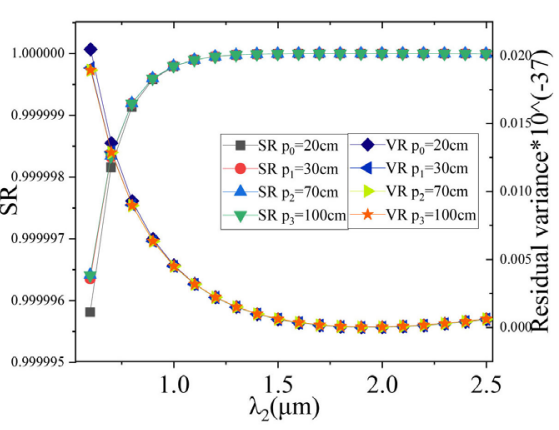

(b)

Figure 6. When $\lambda_{1}$ is $1.5 \mu \mathrm{m}$, the corrected residual variance and the Strehl ratio change after adding the correction coefficient (a) in the case of different internal scales at the same point; (b) when the two points are separated by $\rho$. 
ratio with the wavelength $\lambda_{2}$ are as shown in Figure 6(b). The residual variance at two points of different distances $\rho$ on the aperture of a circle with radius $R$ decreases gradually with the increase of $\lambda_{2}$, the Strehl ratio starts to increase gradually and finally becomes saturated. The corrected residual variance increases with the increase of the distance between point $\rho_{1}$ and $\rho_{2}$ in the wavefront space, indicating that the existence of the correction coefficient makes the corrected residual variance less sensitive to the change of the distance $\rho$ in wavefront space, and it is helpful to improve the quality of wavefront. When compared with the case where the correction coefficient is not added, the quality of the system obviously improves after the correction coefficient is added.

\section{Experimental Verification}

The experiment used a combined beam of wavelengths of $1.55 \mu \mathrm{m}$ and $0.65 \mu \mathrm{m}$ that is emitted horizontally from the same transmitting antenna at a distance of $514 \mathrm{~m}$ from the receiving end. The structure of the receiving end is shown in Figure 7. After the combined beam passes through the optical antenna with a diameter of $250 \mathrm{~mm}$, it is reflected by the deformed mirror into the dichroic beam splitter. The beam of wavelength $0.65 \mu \mathrm{m}$ enters the wavefront sensor A, the beam of wavelength $1.55 \mu \mathrm{m}$ is used as the target correcting beam to enter the wavefront sensor $B$. The adaptive optics system reconstructs the wavefront detected by the wavefront sensor A as a feedback amount and loads it into the deformable mirror correction wavefront through the control box. Both wavefront sensors are relatively calibrated Shack Hartmann wavefront sensors. Figure 8 shows a beam transfer link and the experimental equipment. To avoid the

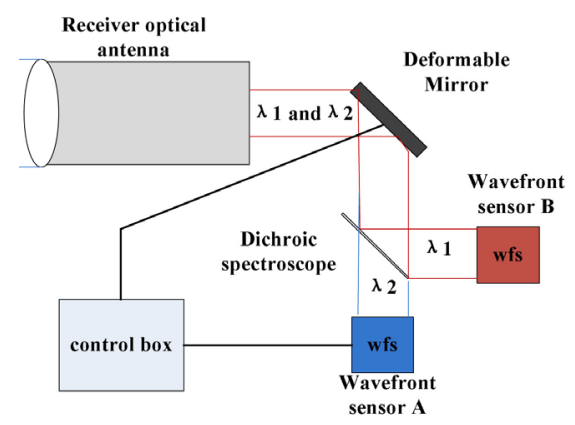

Figure 7. Structure diagram of the receiving end of the adaptive optics system.
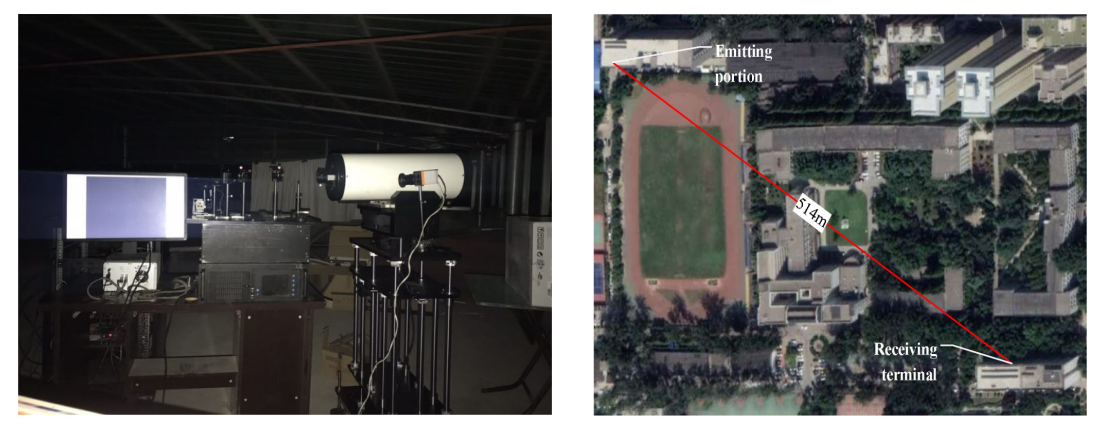

Figure 8. Experimental equipment and link location. 
influence of sunlight the experiment was carried out at night, and to avoid the obstruction of buildings and trees and the vibration caused by the wind on the experimental equipment, the transmitter is placed on the 7th floor of the 5th teaching building of Xi'an University of Science and Technology, and the receiver is placed on the 7th floor of the discipline building. The transmitter and receiver are about $22 \mathrm{~m}$ away from the ground.

When the correction parameters are not added, the system performs a closedloop correction, and the wavefront sensors $\mathrm{A}$ and $\mathrm{B}$ measure the root mean square (RMS) and peak valley (PV) values of the wavefront corresponding to the wavelengths of $1.55 \mu \mathrm{m}$ and $0.65 \mu \mathrm{m}$ respectively after removing the wavefront tilt. As shown in Figure 9, after closed-loop correction the wavefront RMS of the beam with the wavelength of $1.55 \mu \mathrm{m}$ is $0.092-0.413$, the mean value of RMS measured is 0.261 , and the wavefront RMS of the beam with the wavelength of $0.65 \mu \mathrm{m}$ is between 0.34 and 0.62 with a mean value of 0.472 .

The correction coefficient $G$ is calculated from the wavelength according to Equations (17) and (18), the sub-mirror size of the phase-change mirror, and the transmission distance; and is loaded into the control algorithm of the deformable mirror, and then closed-loop correction is performed. Figure 10 shows the

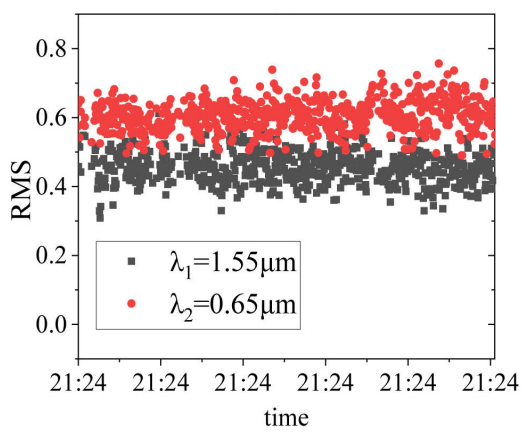

(a)

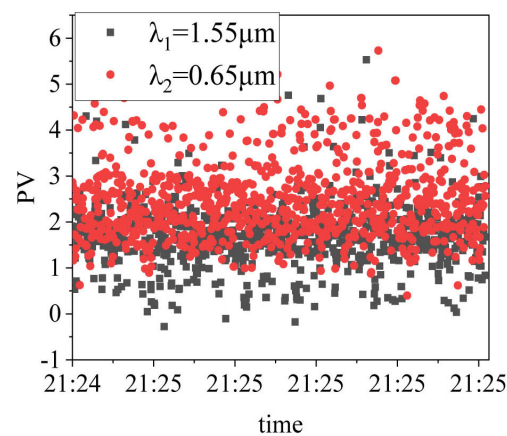

(b)

Figure 9. RMS value and PV value of the two-wavelength wavefront in the closed-loop correction without correction coefficient. (a) RMS value of the two-wavelength wavefront; (b) PV value of the two-wavelength wavefront.

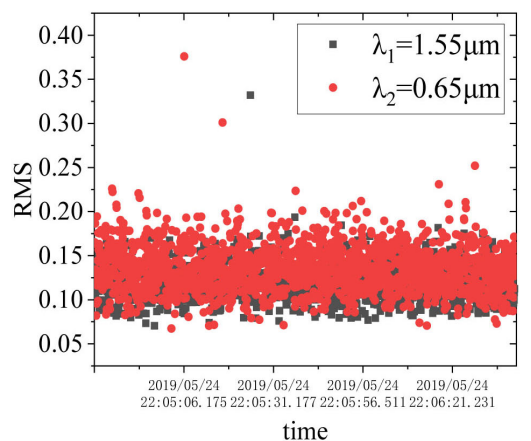

(a)

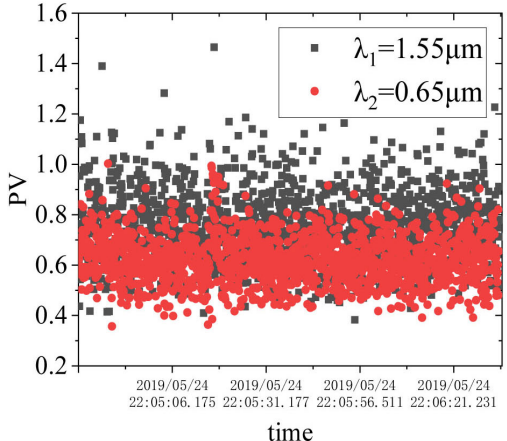

(b)

Figure 10. RMS value and PV value of the two-wavelength wavefront in the closed-loop correction of the correction coefficient. (a) RMS value of the two-wavelength wavefront (b) PV value of the two-wavelength wavefront. 
results of the wavefront sensors A and B again measuring the RMS and PV values of the corresponding wavefront. After closed-loop correction the wavefront RMS of the beam with the wavelength of $1.55 \mu \mathrm{m}$ is between 0.052 and 0.171 , the mean value is 0.121 , and the wavefront RMS of the beam with the wavelength of $0.65 \mu \mathrm{m}$ is between 0.071 and 0.252 with a mean value of 0.153 . Figure 11 shows the Wavelengths measuring $1.5 \mu \mathrm{m}$ and $0.65 \mu \mathrm{m}$ corresponding to a mean of the Zernike coefficient of the wavefront before and after adding correction parameters, the mean value of the first five orders after adding the correction coefficient is lower than that after adding the correction coefficient.

By comparing the correction effect before and after the correction coefficient is added, we find that, after the correction coefficient is added, when the system is closed-loop, the mean value of the wavefront RMS and PV values in the measurement time is smaller than that without the correction coefficient. The wavefront distribution corresponding to $1.55 \mu \mathrm{m}$ after correction is shown in Figure 12(b), and Figure 12(a) shows compared with the case where the correction effect is not added. The correction effect is obviously improved after the correction coefficient is added as shown in Figure 12(b).

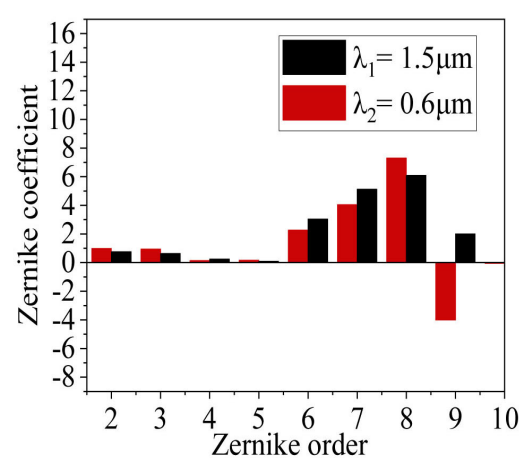

(a)

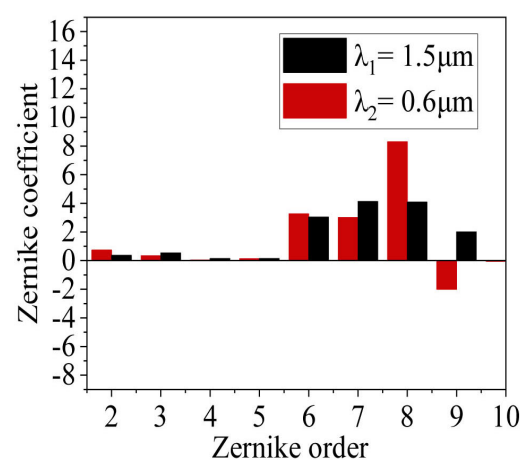

(b)

Figure 11. Wavelengths measuring $1.5 \mu \mathrm{m}$ and $0.65 \mu \mathrm{m}$ corresponding to a mean of the Zernike coefficient of the wavefront (a) before adding correction parameters; and (b) after adding correction parameters.

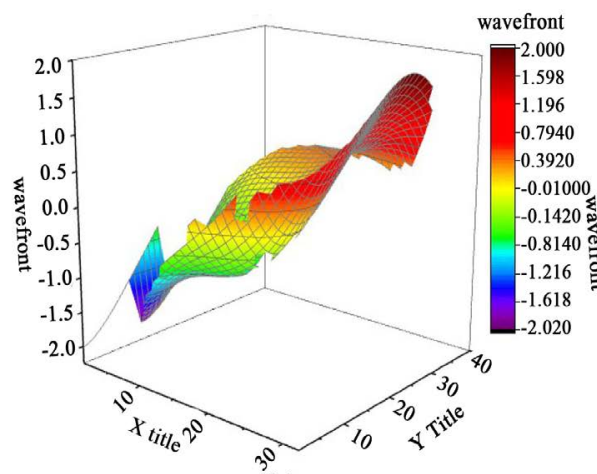

(a)

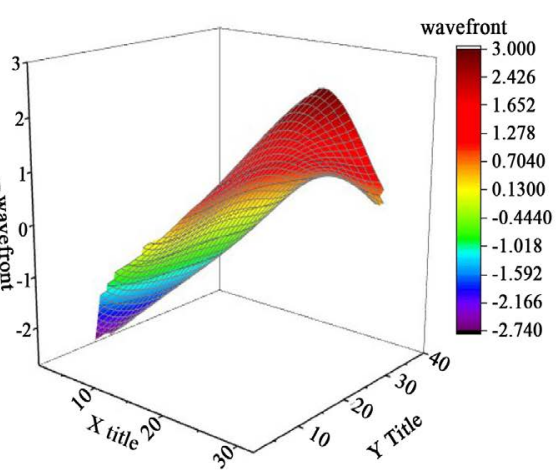

(b)

Figure 12. Wavefront distribution corresponding to $1.5 \mu \mathrm{m}$ wavelength (a) before adding correction parameters (b) after adding correction parameters. 


\section{Conclusion}

When the beam from the laser communication system travels horizontally near the ground, the ensemble average and wavefront correlation function of the wavefront difference corresponding to wavelength can be characterized by the turbulent environmental parameters and the beam wavelength at different minimum correction distances. When the spatial minimum correction distance is zero, the turbulence scale is the main factor for correcting the residual error; when the spatial correction distance is not zero, the spatial minimum correction distance is the main factor for correcting the residual error. In the transmission process, the size of the turbulent internal scale has a more significant effect than the external scale on the wavefront distortion of the wavelength. In the near-surface environment, the number of small-scale turbulent eddies is large, and the turbulent flow has a scale change more rapidly than the outer scale, so the wavefront corresponding to the wavelength in the combined light transmitted horizontally near the ground is more likely to produce spatial differences in the transmission, This makes it easy to drop the quality of the wavefront correction of the adaptive optics system, and thus affects the quality of the laser communication system. The ratio of the wavefront difference corresponding to the wavelength is used as the correction coefficient of the dual-wavelength adaptive optics in the laser communication. The system corrects the value after the beacon wavefront reconstruction and then loads it into the actuator movement amount of the deformable mirror, so that deformed mirror surface type is closer to the conjugate of the signal light's wavefront, thereby improving the corrected Strehl ratio, improving the correction effect and signal to receive power, and improving the information transmission quality of the laser communication system.

\section{Acknowledgements}

We thank LetPub (http://www.letpub.com/) for its linguistic assistance during the preparation of this manuscript.

\section{Funding}

1) Natural Science Foundation of Shaanxi Provincial Department of Education (18JK0341); 2) Xi'an Science and Technology Innovation Guide Project (20110530YD8CG14 (12)); 3) Shaanxi Key Industry Innovation Project (2017 ZDCXL-GY-06-01).

\section{Conflicts of Interest}

The authors declared that they have no conflicts of interest in this work.

\section{References}

[1] Li, X. (2014) Principle and Technology of Star-Ground Laser Communication Link. Telecommunications Science, 30, 46-50+54.

[2] Tyson, R. (1991) Principles of Adaptive Optics. Academic Press, Boston, MA. 
https://doi.org/10.1016/B978-0-12-705900-6.50007-0

[3] Ke, X. and Xi, X. (2004) Introduction to Wireless Laser Communication. Beijing University of Posts and Telecommunications Press, Beijing, 33.

[4] Zhou, R. (1996) Adaptive Optics Theory. Beijing Institute of Technology Press, Beijing, 12.

[5] El-Wakeel, A.S., Mohammed, N.A. and Aly, M.H. (2016) Free Space Optical Communications System Performance under Atmospheric Scattering and Turbulence for 850 and $1550 \mathrm{~nm}$ Operation. Applied Optics, 55, 7276-7286.

https://doi.org/10.1364/AO.55.007276

[6] Rao, R. (2012) Modern Atmospheric Optics. Science Press, Beijing, 370.

[7] Lukin, V.P. and Fortes, B.V. (2001) Adaptive Phase Correction of Turbulent Distortions for Image and Beam under Conditions of Strong Intensity Fluctuations. Proceedings of SPIE-The International Society for Optical Engineering, 4167.

[8] Winocur, J. (1983) Dual-Wavelength Adaptive Optical Systems. Applied Optics, 22, 3711. https://doi.org/10.1364/AO.22.003711

[9] Hogge, C.B. and Butts, R.R. (1982) Effects of Using Different Wavelengths in Wavefront Sensing and Correction. Journal of the Optical Society of America, 72, 606-609. https://doi.org/10.1364/JOSA.72.000606

[10] Lukin, V.P. and Vladimir, P. (1988) Efficiency of a Two-Color Adaptive Optical System. Soviet Journal of Quantum Electronics, 18, 1159-1162. https://doi.org/10.1070/QE1988v018n09ABEH012442

[11] Lukin, V.P. (1979) Efficiency of Some Correction Systems. Optics Letters, 4, 15. https://doi.org/10.1364/OL.4.000015

[12] Ishimaru, A. (1972) Temporal Frequency Spectra of Multifrequency Waves in Turbulent Atmosphere. IEEE Transactions on Antennas and Propagation, 20, 10-19. https://doi.org/10.1109/TAP.1972.1140133

[13] Gorelaya, A.V., Shubenkova, E.V., Dmitriev, D.I., et al. (2015) Investigation of Dual-Wavelength Laser Beam Propagation along the in-Door Atmospheric Path. Optics in Atmospheric Propagation \& Adaptive Systems XVIII, International Society for Optics and Photonics. https://doi.org/10.1117/12.2194332

[14] Lee, R.W. and Harp, J.C. (1969) Weak Scattering in Random Media, with Applications to Remote Probing. Proceedings of the IEEE, 57, 375-406. https://doi.org/10.1109/PROC.1969.6993

[15] Feng, Y., Song, Z. and Gong, Z. (1991) Atmospheric Dispersion Effect in Adaptive Optics. Journal of Infrared and Millimeter Waves, No. 3, 217-221.

[16] Ishimaru, A. (1978) Wave Propagation and Scattering in Random Media. In: Wave Propagation and Scattering in Random Media, IEEE Press, New York.

[17] Wu, J., Yang, C. and Liu, J. (2005) Theory of Optical Transmission in the Atmosphere. Beijing University of Posts and Telecommunications Press, Beijing, 162.

[18] Yuan, R. and Zeng, Z. (2000) Comparison of Measurement of Refractive Index Structure Constants by Different Methods. Acta Optica Sinica, 20, 755-761.

[19] Tyson, R.K. and Wizinowich, P.L. (1992) Principles of Adaptive Optics. Physics Today, 45, 100. https://doi.org/10.1063/1.2809540

[20] Li, Y., Qiang, X., Zong, F., et al. (2010) Effect of Turbulence Internal Scale on the Fluctuation Spectrum of Light Wave. Intense Laser and Particle Beam, 22, 2276-2280. https://doi.org/10.3788/HPLPB20102210.2276 